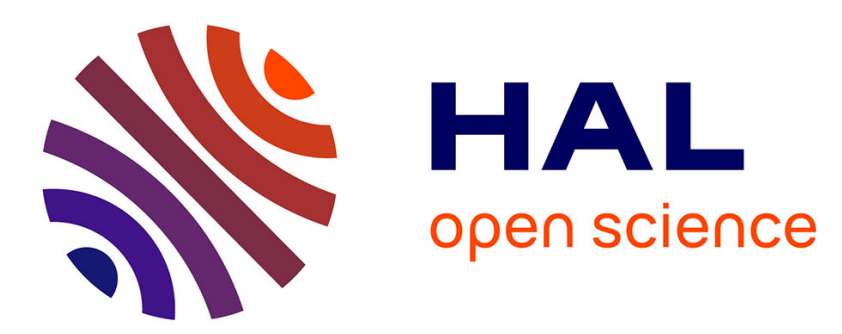

\title{
Gradient estimates for elliptic regularizations of semilinear parabolic and degenerate elliptic equations
}

Henri Berestycki, Francois Hamel

\section{To cite this version:}

Henri Berestycki, Francois Hamel. Gradient estimates for elliptic regularizations of semilinear parabolic and degenerate elliptic equations. Communications in Partial Differential Equations, 2005, pp.139. hal-00003738

\section{HAL Id: hal-00003738 \\ https://hal.science/hal-00003738}

Submitted on 2 Jan 2005

HAL is a multi-disciplinary open access archive for the deposit and dissemination of scientific research documents, whether they are published or not. The documents may come from teaching and research institutions in France or abroad, or from public or private research centers.
L'archive ouverte pluridisciplinaire HAL, est destinée au dépôt et à la diffusion de documents scientifiques de niveau recherche, publiés ou non, émanant des établissements d'enseignement et de recherche français ou étrangers, des laboratoires publics ou privés. 


\title{
Gradient estimates for elliptic regularizations of semilinear parabolic and degenerate elliptic equations
}

\author{
Henri Berestycki * $\quad$ François Hamel ${ }^{\dagger}$
}

\begin{abstract}
This article is chiefly concerned with elliptic regularizations of semilinear parabolic equations of the type

$$
\varepsilon u_{t t}-u_{t}+L u+f(u)=0
$$

where $L$ is an elliptic operator in the space variables $x$. We establish $L^{\infty}$ gradient estimates up to the boundary which are uniform with respect to the small elliptic regularization parameter $\varepsilon$. Such estimates were used for instance in proving the existence of pulsating travelling front solutions for reaction-diffusion equations in a previous work [2].

Similar $x$-gradient estimates are also obtained, both in the interior of the domain and up to the boundary, for elliptic (in $(x, y)$ variables) regularizations$$
L_{x} u+\varepsilon L_{x y} u+\beta(x, y) \cdot \nabla_{x, y} u+f(x, y, u)=0
$$

of degenerate elliptic equations.

\section{Introduction and main results}

The aim of this paper is to state some $L^{\infty}$ gradient estimates which are uniform with respect to some small parameter $\varepsilon$. We are concerned here with bounded solutions of some regularizing elliptic approximations of parabolic equations or more general degenerate elliptic equations.

To illustrate our results on a simple case, consider the semilinear parabolic equation :

$$
u_{t}=\Delta_{x} u+f(u)
$$

set in a domain (in $(t, x)$ variables) $\Omega \subset \mathbb{R} \times \mathbb{R}^{N}$. Associated with this equation, we can define a family of elliptic equations :

$$
\varepsilon u_{t t}-u_{t}+\Delta_{x} u+f(u)=0
$$

For each $\varepsilon>0$, this equation is elliptic in all the variables $X=(t, x)$.

\footnotetext{
*EHESS, CAMS, 54 Boulevard Raspail, F-75006 Paris, France. hb@ehess.fr

${ }^{\dagger}$ Université Aix-Marseille III, LATP, Faculté Saint-Jérôme, Avenue Escadrille Normandie-Niemen, F-13397 Marseille Cedex 20, France. francois.hamel@univ.u-3mrs.fr
} 
Problem (1.2) formally degenerates to a parabolic equation as $\varepsilon \rightarrow 0^{+}$. The problem we investigate here is concerned with obtaining uniform gradient estimates for the solutions of (1.2), uniformly with respect to $\varepsilon$, say $\varepsilon \in[0,1]$. Actually, gradient estimates can be obtained from classical parabolic theory for $\varepsilon=0$ or from classical elliptic theory for any given $\varepsilon_{0}>0$. But the classical elliptic a priori estimates blow up when $\varepsilon \rightarrow 0^{+}$. The purpose of this paper is to derive some estimates which are uniform with respect to $\varepsilon \in[0,1]$. Such a priori estimates are needed in order to pass to the limit $\varepsilon \rightarrow 0^{+}$for solutions of equations of type (1.2).

More precisely, consider a sequence of classical solutions $u^{\varepsilon}$ of (1.2). Under some boundary conditions on $\partial \Omega$ for the functions $u^{\varepsilon}$, uniform $H^{1}$ estimates can easily be derived by integrating equation (1.2) or by multiplying it by $u^{\varepsilon}$ or $u_{t}^{\varepsilon}$ (see e.g. Lions [11] or Lions and Magenes [12]). In some situations, stronger pointwise estimates are required. For instance, this is the case when one wants to pass to the limit in some quantities like $\max _{K} u^{\varepsilon}$ where $K$ is a compact subset of $\bar{\Omega}$.

Such a case occured in [2], where elliptic regularizations of the type (1.2) were used in the analysis of pulsating travelling fronts for a class of parabolic reaction-diffusion-advection equations in periodic domains. Actually, equations more general than (1.1) or (1.2) were dealt with in [2]. To make sure that the limiting function as $\varepsilon \rightarrow 0^{+}$is not trivial and satisfies some a priori upper bounds, a normalization of the type $\max _{K} u^{\varepsilon}=a$ was used, for some constant $a$ independent of $\varepsilon$ and for some compact set $K$. Uniform gradient estimates of the type (1.5) or (1.7) below then allowed us to pass to the limit $\varepsilon \rightarrow 0^{+}$.

Let us also mention that Heinze [7] and Xin [14] used similar limiting procedures for equations in the whole space, for which estimates of this type are useful.

Regularization techniques are a very classical approach and uniform gradient estimates have also been derived in other contexts. Let us mention for instance regularizing approximations of free boundary problems with singular reaction terms [1], [3], or the viscous approximations of some models in fluid mechanics [4], [5].

Theorem 1.6 below states some $x$-gradient estimates for bounded solutions of a more general class of elliptic regularizations. There we study degenerate equations which are elliptic with respect to the $N_{1}$ first variables $x=\left(x_{1}, \cdots, x_{N_{1}}\right)\left(1 \leq N_{1}<N_{2}\right)$ and which are degenerate in the $N_{2}-N_{1}$ other variables $y=\left(x_{N_{1}+1}, \cdots, x_{N_{2}}\right)$. Although the class of elliptic equations dealt with in Theorem 1.6 is more general than these in Theorems 1.1, 1.2 and 1.3, we choose to state Theorems 1.1, 1.2 and 1.3 first. The latter are concerned with elliptic regularizations of parabolic equations. Theorems 1.2 and 1.3 below are devoted to some uniform pointwise gradient estimates for bounded solutions of a more general class than (1.2) of elliptic regularizations of parabolic equations. Both interior and boundary estimates for the gradients in $x$ variables are derived. However, to illustrate our results, we first state Theorem 1.1 on interior $x$-gradient estimates in the case of problem (1.2).

In what follows, for any $d$ and $\xi \in \mathbb{R}^{d},|\xi|$ denotes the euclidian norm of $\xi:|\xi|=$ $\sqrt{\xi_{1}^{2}+\cdots+\xi_{d}^{2}}$. For any $r>0, B_{r}(\xi)$ denotes the open ball with center $\xi$ and radius $r$. For any sufficiently smooth function $v$ defined in a set $D \subset \mathbb{R}^{N+1}$, with the generic notation $\left(t, x_{1}, \cdots, x_{N}\right)$ for the points of $\mathbb{R}^{N+1}$, we set $v_{t}=\partial_{t} v, v_{i}=\partial_{x_{i}} v$, for $1 \leq i \leq N$, and call $\operatorname{osc}_{D}(v)=\sup _{D} v-\inf _{D} v$ (the oscillation of $v$ in $D$ ) if $v$ is bounded in $D$. We also use the usual summation convention for the partial derivatives. Lastly, $d(X, D)$ denotes the euclidian 
distance of a point $X$ to the set $D$, under the convention that $d(X, D)=+\infty$ if $D=\emptyset$.

Theorem 1.1 (Interior estimates for problem (1.2)) Let $\Omega$ be an open subset of $\mathbb{R} \times \mathbb{R}^{N}$. Let $f$ be a $C^{1}(\mathbb{R})$ function, with bounded derivative.

Let $0 \leq \varepsilon \leq 1$ and let $u$ be a solution of class $C^{3}(\Omega) \cap L_{l o c}^{\infty}(\Omega)$ of equation (1.2) in $\Omega$.

Then, for all $X \in \Omega$,

$$
\left|\nabla_{x} u(X)\right|^{2} \leq C \times\left(1+\frac{1}{d(X, \partial \Omega)^{2}}\right),
$$

where

$$
C=C_{0} \times\|u\|_{L^{\infty}\left(B_{X}\right)} \times\left(\operatorname{osc}_{B_{X}}(u)+\|f\|_{L^{\infty}\left(\left[m_{X}, M_{X}\right]\right)}\right),
$$

$B_{X}=B_{d(X, \partial \Omega) / 2}(X), m_{X}=\inf _{B_{X}} u$ and $M_{X}=\sup _{B_{X}} u$. The constant $C_{0}=C_{0}\left(N,\left\|f^{\prime}\right\|_{L^{\infty}(\mathbb{R})}\right)$ only depends on $N$ and $\left\|f^{\prime}\right\|_{L^{\infty}(\mathbb{R})}$.

The following theorem deals with interior $x$-gradient estimates in the case of a elliptic regularizations of more general parabolic equations :

Theorem 1.2 (Interior estimates for elliptic regularizations of parabolic equations) Let $\Omega$ be an open subset of $\mathbb{R} \times \mathbb{R}^{N}$. Let $\left(\alpha^{i j}\right)_{1 \leq i, j \leq N}$ be a $C^{1}(\bar{\Omega})$ symmetric matrix field such that there exists $\sigma>0$ with

$$
\forall(X, \xi) \in \bar{\Omega} \times \mathbb{R}^{N}, \quad \sum_{1 \leq i, j \leq N} \alpha^{i j}(X) \xi_{i} \xi_{j} \geq \sigma|\xi|^{2} .
$$

Let $\left(\beta^{i}\right)_{1 \leq i \leq N}$ be a $C^{1}(\bar{\Omega})$ vector field and $f=f(X, u)$ be a $C_{l o c}^{1}(\Omega \times \mathbb{R})$ function such that $\partial_{u} f$ is bounded in $\Omega \times \mathbb{R}$. Let $b \geq 0$ be such that, for all $1 \leq i, j \leq N,\left\|\alpha^{i j}\right\|_{C^{1}(\bar{\Omega})}+\left\|\beta^{i}\right\|_{C^{1}(\bar{\Omega})}+$ $\left\|\partial_{u} f\right\|_{L^{\infty}(\Omega \times \mathbb{R})} \leq b$.

Let $0 \leq \varepsilon \leq 1$ and let $u$ be a solution of class $C^{3}(\Omega) \cap L_{l o c}^{\infty}(\Omega)$ of the equation

$$
\varepsilon u_{t t}-u_{t}+\alpha^{i j}(X) u_{i j}+\beta^{i}(X) u_{i}+f(X, u)=0 \text { in } \Omega .
$$

Then, for all $X \in \Omega$,

$$
\left|\nabla_{x} u(X)\right|^{2} \leq C \times\left(1+\frac{1}{d(X, \partial \Omega)^{2}}\right)
$$

where

$$
C=C_{1} \times\left[\|u\|_{L^{\infty}\left(B_{X}\right)}\left(\operatorname{osc}_{B_{X}}(u)+\|f\|_{L^{\infty}\left(B_{X} \times\left[m_{X}, M_{X}\right]\right)}\right)+\left\|\nabla_{x} f\right\|_{L^{\infty}\left(B_{X} \times\left[m_{X}, M_{X}\right]\right)}^{2}\right],
$$

$B_{X}=B_{d(X, \partial \Omega) / 2}(X), m_{X}=\inf _{B_{X}} u$ and $M_{X}=\sup _{B_{X}} u$. The constant $C_{1}=C_{1}(N, \sigma, b)$ only depends on $N, \sigma$ and $b$.

Theorem 1.3 below is devoted to $x$-gradient estimates up to the boundary for the same class of problems (1.4) : 
Theorem 1.3 (Estimates up to the boundary for elliptic regularizations of parabolic equations) Under the conditions of Theorem 1.2, let $\Sigma$ be a smooth (at least globally $C^{3}$ ) subset of $\partial \Omega$ and assume that, at each point $X \in \Sigma$, the unit normal $\nu=\nu(X)$ to $\Omega$ exists and that its t-component is zero. Assume that for each $X \in \Sigma$, there exists $r_{X}>0$ such that $\partial \Omega \cap B_{r_{X}}(X) \subset \Sigma$. Let $\mu$ be a $C^{3}(\Sigma)$ unit vector field whose $t$-component is zero and assume that there exists $\gamma>0$ such that $\mu(X) \cdot \nu(X) \geq \gamma>0$ for all $X \in \Sigma$.

For each $X \in \partial \Omega$ and each $\delta>0$, let $\Gamma_{X, \delta}$ denote the connected component of $\partial \Omega \cap$ $B_{\delta}(X)$ containing $X$. Assume that there exists $\eta>0$ such that, for all $X \in \Sigma$, the connected component of $B_{\eta}(X) \backslash \Gamma_{X, \eta}$ containing $X-r \nu(X)$ for $r>0$ small enough is included in $\Omega$. Assume also that the function $f$ is in $C_{l o c}^{1}((\Omega \cup \Sigma) \times \mathbb{R})$.

Let $0 \leq \varepsilon \leq 1$ and let $u$ be a solution of class $C^{2}(\Omega \cup \Sigma) \cap C^{3}(\Omega) \cap L_{l o c}^{\infty}(\Omega)$ of the equation

$$
\left\{\begin{array}{rll}
\varepsilon u_{t t}-u_{t}+\alpha^{i j}(X) u_{i j}+\beta^{i}(X) u_{i}+f(X, u) & =0 & \text { in } \Omega \\
\mu \cdot \nabla_{X} u & =0 & \text { on } \Sigma
\end{array}\right.
$$

Then, for all $X \in \Omega \cup \Sigma$,

$$
\left|\nabla_{x} u(X)\right|^{2} \leq C \times\left(1+\frac{1}{d(X, \partial \Omega \backslash \Sigma)^{2}}\right)
$$

where

$C=C_{2} \times\left[\|u\|_{L^{\infty}\left(B_{X} \cap \Omega\right)}\left(\operatorname{osc}_{B_{X} \cap \Omega}(u)+\|f\|_{L^{\infty}\left(B_{X} \cap \Omega \times\left[m_{X}, M_{X}\right]\right)}\right)+\left\|\nabla_{x} f\right\|_{L^{\infty}\left(B_{X} \cap \Omega \times\left[m_{X}, M_{X}\right]\right)}^{2}\right]$,

$B_{X}=B_{d(X, \partial \Omega \backslash \Sigma) / 2}(X), m_{X}=\inf _{B_{X} \cap \Omega} u$ and $M_{X}=\sup _{B_{X} \cap \Omega} u$. The constant $C_{2}=$ $C_{2}(N, \sigma, b, \eta, \gamma, \mu, \Sigma)$ only depends on $N, \sigma, b, \eta, \gamma,\|\mu\|_{C^{3}(\Sigma)}$ and on the bounds of the derivatives up to the third order of the functions representing $\Sigma$.

Remark 1.4 Theorem 1.3 is clearly stronger than Theorem 1.2, which corresponds to the case where $\Sigma=\emptyset$. But for the sake of clarity we chose to write two different results for the interior estimates and for the estimates up to the boundary.

Remark 1.5 Let us mention now some special cases of applications of the above Theorems 1.1, 1.2 and 1.3 .

The first and simplest case is when the function $u$ is constant. In this case $\nabla_{x} u=u_{t}=0$, whence $f(X, u)=\nabla_{x} f(X, u)=0$ in $\Omega,{ }^{1}$ and the right hand sides of inequalities (1.3), (1.5) or (1.7) are equal to 0.

Assume now that $\left(u^{\varepsilon}\right)_{0 \leq \varepsilon \leq 1}$ is a family, bounded in $L^{\infty}(\Omega)$, of solutions of class $C^{2}(\Omega \cup$ $\Sigma) \cap C^{3}(\Omega)$ of equations of the type (1.2), (1.4) or (1.6), with some coefficients $\alpha^{\varepsilon}, \beta^{\varepsilon}, f^{\varepsilon}$ or $\mu^{\varepsilon}$ such that the hypotheses of the above theorems are valid for some $\sigma, b$ and $\gamma$ uniformly in $\varepsilon$,-in case where such assumptions make sense, according to which problem (1.2), (1.4) or (1.6) is concerned-and assume that $\sup _{0 \leq \varepsilon<1}\left\|\mu^{\varepsilon}\right\|_{C^{3}(\Sigma)}<\infty$ and that the family $\left(f^{\varepsilon}\right)_{0 \leq \varepsilon \leq 1}$ is bounded in $C^{1}(\bar{\Omega} \times \mathbb{R})$ (this is the case if there is only one such function $f$ ). Then the above

\footnotetext{
${ }^{1}$ in case of problem $(1.2)$, one then has $f(u)=0$ in $\Omega$
} 
theorems say that the family $\left(u^{\varepsilon}\right)_{0 \leq \varepsilon \leq 1}$ is bounded in $C_{l o c}^{1}(\Omega \cup \Sigma)$ with respect to $x$, namely, for each compact set $K \subset \Omega \cup \Sigma$, there exists $A_{K} \geq 0$ such that

$$
\forall 0 \leq \varepsilon \leq 1, \quad\left\|\nabla_{x} u^{\varepsilon}\right\|_{L^{\infty}(K)} \leq A_{K} .
$$

In particular, if $\Omega$ is a straight infinite cylinder of the type $\mathbb{R} \times D$, where $D$ is a $C^{3}$ bounded domain of $\mathbb{R}^{N}$, and if $\Sigma=\partial \Omega$, then $\left|\nabla_{x} u^{\varepsilon}(X)\right|$ is bounded uniformly with respect to $X \in \bar{\Omega}$ and independently of $\varepsilon$. This case occured in our previous paper [2]. Gradient estimates of the type (1.7) allowed us to pass to the limit in some quantities like $\max _{K} u^{\varepsilon}$, where $K$ was a fixed compact subset of $\bar{\Omega}$.

For similar problems with $C^{1}$ source terms $f(X)$, interior estimates involving the $C^{1}$ norm of $f$ have been obtained by Lieberman [10]. Similar estimates up the boundary involving the $C^{2}$ bound of the trace of $u$ on the boundary have also been derived in [10]. Our point of view here is different since the nonlinear term $f$ depends on $X$ as well as on $u$ and, therefore, the $C^{1}$ bound of the function $X \mapsto f(X, u(X))$ is not given. Furthermore, no assumption is made here on the $C^{2}$ bound of the trace of $u$ on the boundary.

As already emphasized, when, say, $\varepsilon=1$, the gradient estimates (1.3), (1.5) or (1.7) are particular cases of classical elliptic estimates (see e.g. [6]). But, as far as we know, for regularizing problems of the type (1.2), (1.4) or (1.6), either interior or boundary estimates have not been yet obtained uniformly with respect to small $\varepsilon$.

Further on, a parabolic equation can also be viewed as a degenerate elliptic equation, which is elliptic in $x$ and degenerate in $t$. It is then natural to wonder whether similar gradient estimates hold for more general degenerate elliptic equations. More precisely, the following Theorem 1.6 is concerned with some $L^{\infty}$ gradient estimates with respect to a set of variables $x$, for elliptic regularizations of equations which are elliptic in $x$ and degenerate in another set of variables $y$.

In what follows, for $1 \leq N_{1}<N_{2}$, we use the generic notation

$$
X=(x, y), \quad x=\left(x_{1}, \cdots, x_{N_{1}}\right), y=\left(x_{N_{1}+1}, \cdots, x_{N_{2}}\right)
$$

for the points in $\mathbb{R}^{N_{2}}$, and we write

$$
|\xi|_{1}^{2}=\sum_{i=1}^{N_{1}}\left|\xi_{i}\right|^{2}, \quad|\xi|_{2}^{2}=\sum_{l=N_{1}+1}^{N_{2}}\left|\xi_{l}\right|^{2}
$$

for all $\xi \in \mathbb{R}^{N_{2}}$. For any sufficiently smooth function $v$ defined in a set $D \subset \mathbb{R}^{N_{2}}$, we use the notations $v_{i}=\partial_{x_{i}} v, v_{j}=\partial_{x_{j}} v$ and $v_{k}=\partial_{x_{k}} v$ for the partial derivatives of $v$ with respect to the variables $x_{i}$ and $x_{j}$, with $1 \leq i, j, k \leq N_{1}$, and $v_{l}=\partial_{x_{l}} v$ and $v_{m}=\partial_{x_{m}} v$ for the partial derivatives of $v$ with respect to the variables $x_{l}$ and $x_{m}$, with $N_{1}+1 \leq l, m \leq N_{2}$. We use the same type of notations for the higher-order derivatives. For instance, the notation $v_{i l}=\partial_{x_{i} x_{l}}^{2} v$ means the partial derivative of $v$ with respect to the variables $x_{i}$ and $x_{l}$, with $1 \leq i \leq N_{1}$ and $N_{1}+1 \leq l \leq N_{2}$. More generally speaking, we use the notation $v_{I}=\partial_{x_{I}} v$ for the partial derivative of $v$ with respect to the variable $x_{I}$, with $1 \leq I \leq N_{2}$. We also use the usual summation convention for the partial derivatives. 
Theorem 1.6 (Interior and boundary estimates for elliptic regularizations of more general degenerate equations) Let $1 \leq N_{1}<N_{2}$ and let $\Omega$ be an open subset of $\mathbb{R}^{N_{2}}$. Let $\left(\alpha^{I J}\right)_{1 \leq I, J \leq N_{2}}$ be a $C^{1}(\bar{\Omega})$ symmetric matrix field and assume there exist $\sigma_{1}>0$ and $\sigma_{2}>0$ with

$$
\sum_{1 \leq i, j \leq N_{1}} \alpha^{i j}(X) \xi_{i} \xi_{j} \geq \sigma_{1}|\xi|_{1}^{2}, \quad \sum_{N_{1}+1 \leq l, m \leq N_{2}} \alpha^{l m}(X) \xi_{l} \xi_{m} \geq \sigma_{2}|\xi|_{2}^{2}
$$

for all $(X, \xi) \in \bar{\Omega} \times \mathbb{R}^{N}$. Let $\left(\beta^{I}\right)_{1 \leq I \leq N_{2}}$ be a $C^{1}(\bar{\Omega})$ vector field and $b \geq 0$ be such that, for all $1 \leq I, J \leq N_{2},\left\|\alpha^{I J}\right\|_{C^{1}(\bar{\Omega})}+\left\|\beta^{\bar{I}}\right\|_{C^{1}(\bar{\Omega})} \leq b$. Assume also that $\alpha_{k}^{l m}=\beta_{k}^{l}=0$ in $\bar{\Omega}$ for all $1 \leq k \leq N_{1}$ and $N_{1}+1 \leq l, m \leq N_{2}$.

Let $\Sigma$ be a smooth (at least globally $C^{3}$ ) subset of $\partial \Omega$ and assume that, at each point $X \in \Sigma$, the unit normal $\nu=\nu(X)$ to $\Omega$ exists and that its $y$-components are zero. Assume that for each $X \in \Sigma$, there exists $r_{X}>0$ such that $\partial \Omega \cap B_{r_{X}}(X) \subset \Sigma$. Let $\mu$ be a $C^{3}(\Sigma)$ unit vector field whose $y$-components are zero and assume that there exists $\gamma>0$ such that $\mu(X) \cdot \nu(X) \geq \gamma>0$ for all $X \in \Sigma$. Assume moreover that there exists $\eta>0$ such that, for all $X \in \Sigma$, the connected component of $B_{\eta}(X) \backslash \Gamma_{X, \eta}$ containing $X-r \nu(X)$ for $r>0$ small enough is included in $\Omega$, where $\Gamma_{X, \eta}$ has the same definition as in Theorem 1.3.

Let $f=f(X, u)$ be a $C_{l o c}^{1}(\Omega \cup \Sigma \times \mathbb{R})$ function such that $\partial_{u} f$ is bounded in $\Omega \times \mathbb{R}$ and $\left\|\partial_{u} f\right\|_{L^{\infty}(\Omega \times \mathbb{R})} \leq b$. Let $0 \leq \varepsilon \leq 1$ and let $u$ be a solution of class $C^{2}(\Omega \cup \Sigma) \cap C^{3}(\Omega) \cap L_{\text {loc }}^{\infty}(\Omega \cup \Sigma)$ of the equation

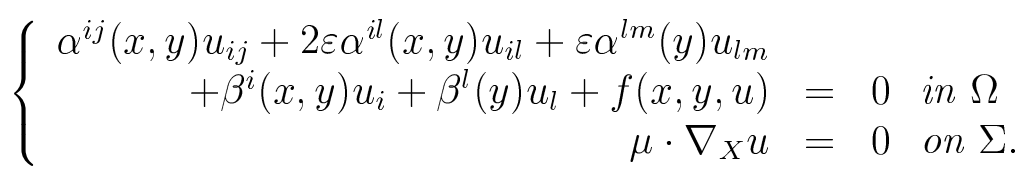

Then there exists $\tilde{\varepsilon}_{0}=\tilde{\varepsilon}_{0}\left(N_{1}, N_{2}, \sigma_{1}, \sigma_{2}, b, \gamma, \mu, \Sigma\right) \in(0,1]$ such that if $0 \leq \varepsilon \leq \tilde{\varepsilon}_{0}$,

$$
\left|\nabla_{x} u(X)\right|^{2} \leq C \times\left(1+\frac{1}{d(X, \partial \Omega \backslash \Sigma)^{2}}\right)
$$

for all $X \in \Omega \cup \Sigma$, where

$$
C=C_{3} \times\left[\|u\|_{L^{\infty}\left(B_{X} \cap \Omega\right)}\left(\operatorname{osc}_{B_{X} \cap \Omega}(u)+\|f\|_{L^{\infty}\left(B_{X} \cap \Omega \times\left[m_{X}, M_{X}\right]\right)}\right)+\left\|\nabla_{x} f\right\|_{L^{\infty}\left(B_{X} \cap \Omega \times\left[m_{X}, M_{X}\right]\right)}^{2}\right],
$$

$B_{X}=B_{d(X, \partial \Omega \backslash \Sigma) / 2}(X), m_{X}=\inf _{B_{X} \cap \Omega} u$ and $M_{X}=\sup _{B_{X} \cap \Omega} u$. The constant $C_{3}=$ $C_{3}\left(N_{1}, N_{2}, \sigma_{1}, \sigma_{2}, b, \eta, \gamma, \mu, \Sigma\right)$ only depends on $N_{1}, N_{2}, \sigma_{1}, \sigma_{2}, b, \eta, \gamma,\|\mu\|_{C^{3}(\Sigma)}$ and on the bounds of the derivatives up to the third order of the functions representing $\Sigma$.

Theorem 1.6 is clearly stronger than Theorems 1.1, 1.2 and 1.3, the latter corresponding to the case $N_{2}=N_{1}+1$ and $t=y=x_{N_{2}}$.

Notice that the approximating operator in equation (1.9) involves cross derivatives of the type $u_{i l}$ with $1 \leq i \leq N_{1}$ and $N_{1}+1 \leq l \leq N_{2}$. The operator acting on the $x$-variables is elliptic. The operator acting on the $y$-variables is elliptic as well, but its ellipticity constant is proportional to $\varepsilon$ (note here that for some technical reasons the coefficients $\alpha^{l m}$ and $\beta^{l}$ of the elliptic regularization are assumed to depend only on the $y$-variables). It follows from the proof of Theorem 1.6 (see Lemma 2.1 below) that the operator in equation (1.9), acting on 
all the $(x, y)$-variables, is actually elliptic for $\varepsilon$ small enough, with ellipticity constant being proportional to $\varepsilon$. For the same reason, $\tilde{\varepsilon}_{0}$ can be chosen equal to 1 in the case where there is no cross second-order partial derivatives, namely if $\alpha^{i l}=0$ for all $1 \leq i \leq N_{1}$ and $N_{1}+1 \leq l \leq N_{2}$. This is especially the case in Theorems 1.1, 1.2 or 1.3.

Remark 1.7 Estimates for the gradient of $u$ with respect to the $y$-variables do not hold in general. For instance, in $\Omega=\mathbb{R}^{2}$, the functions $u^{\varepsilon}(x, y)=\sin (y / \sqrt{\varepsilon})$ (for $\varepsilon>0$ ) satisfy the equations $u_{x x}^{\varepsilon}+\varepsilon u_{y y}^{\varepsilon}+u^{\varepsilon}=0$ but, say, $\left|u_{y}^{\varepsilon}(0,0)\right|$ is not bounded uniformly with respect to $\varepsilon \in(0,1]$.

On the other hand, the estimates (1.10) do not hold in general for large $\varepsilon$. For instance, in $\Omega=\{x \in \mathbb{R}, y<0\}$, the functions $u^{\varepsilon}(x, y)=\sin (\sqrt{\varepsilon} x) e^{y}$ are bounded solutions of the equations $u_{x x}^{\varepsilon}+\varepsilon u_{y y}^{\varepsilon}=0$ but do not satisfy (1.10) uniformly with respect to $\varepsilon \geq 1$.

Theorem 1.6 is concerned with both interior and up to the boundary estimates. Let us just mention here a corollary about the uniform interior estimates for a family of solutions of equations of the type (1.9).

Corollary 1.8 Under the assumptions of Theorem 1.6, let $\left(u^{\varepsilon}\right)_{0 \leq \varepsilon \leq 1}$ be a family, bounded in $L^{\infty}(\Omega)$, of solutions of equations of type (1.9) in $\Omega \subset \mathbb{R}^{N_{2}}$, for some coefficients $\alpha^{\varepsilon}$, $\beta^{\varepsilon}$, $f^{\varepsilon}$ or $\mu^{\varepsilon}$ such that the hypotheses of Theorem 1.6 are valid for some $\sigma_{1}, \sigma_{2}, b$ and $\gamma$ uniformly in

$\varepsilon$, and assume that $\sup _{0 \leq \varepsilon \leq 1}\left\|\mu^{\varepsilon}\right\|_{C^{3}(\Sigma)}<\infty$ and that the families $\left(f^{\varepsilon}\right)_{0 \leq \varepsilon \leq 1},\left(\partial_{u} f^{\varepsilon}\right)_{0 \leq \varepsilon \leq 1}$ and $\left(\nabla_{x} f^{\varepsilon}\right)_{0 \leq \varepsilon \leq 1}$ are bounded in $L^{\infty}(\bar{\Omega} \times \mathbb{R}$ ) (this is the case if there is only one such function $f$ bounded in $\Omega \times \mathbb{R}$ together with its derivatives). Then, for some small enough $\tilde{\varepsilon}_{0}>0$, the family $\left(\nabla_{x} u^{\varepsilon}\right)_{0 \leq \varepsilon \leq \tilde{\varepsilon}_{0}}$ is bounded in $L_{\text {loc }}^{\infty}(\Omega \cup \Sigma)$.

Remark 1.9 Under the notations of Theorem 1.6, if the function $u$ as well as the coefficients of the equation (1.9) are smoother, then estimates of the type (1.10) for the higher-order derivatives $\left|\partial^{n_{1}+\cdots+n_{N_{1}}} u / \partial x_{1}^{n_{1}} \cdots \partial x_{N_{1}}^{n_{N_{1}}}\right|^{2}$ of $u$ could be obtained, where $d(X, \partial \Omega \backslash \Sigma)^{2}$ is replaced with $d(X, \partial \Omega \backslash \Sigma)^{2\left(n_{1}+\cdots+n_{N_{1}}\right)}$.

The proofs of Theorems 1.2 and 1.6 are based on the maximum principle applied to Bernstein-type functions involving $\left|\nabla_{x} u\right|^{2}$ and $u^{2}$, following the ideas of Ladyzenskaja, Solonnikov and Ural'Ceva [8] for parabolic equations. Similar techniques had been applied for non-degenerating elliptic equations, see e.g. Ladyzenskaja, Ural'Ceva [9] and Sperb [13].

\section{Proof of the theorems}

Since the other results are particular cases of Theorem 1.6, we give directly the proof of the general case of Theorem 1.6.

Before going into the proof of Theorem 1.6, let us prove the following elementary lemma, which states that the operator in equation (1.9) is actually elliptic in all $(x, y)$-variables, with ellipticity constant being proportional to $\varepsilon$, for $\varepsilon$ small enough. 
Lemma 2.1 Under the assumptions of Theorem 1.6, call $\bar{\alpha}^{I J}=\alpha^{I J}$ if $1 \leq I, J \leq N_{1}$, and $\bar{\alpha}^{I J}=\varepsilon \alpha^{I J}$ if $1 \leq I, J \leq N_{2}$ and $I$ or $J$ is greater than $N_{1}$. There exists $\varepsilon_{0}=$ $\varepsilon_{0}\left(N_{1}, N_{2}, \sigma_{1}, \sigma_{2}, b\right) \in(0,1]$ such that, if $0 \leq \varepsilon \leq \varepsilon_{0}$, then

$$
\sum_{1 \leq I, J \leq N_{2}} \bar{\alpha}^{I J}(X) \xi_{I} \xi_{J} \geq \frac{\sigma_{1}}{2}|\xi|_{1}^{2}+\varepsilon \frac{\sigma_{2}}{2}|\xi|_{2}^{2}
$$

for $\operatorname{all}(X, \xi) \in \bar{\Omega} \times \mathbb{R}^{N}$.

Proof. Let $(X, \xi) \in \bar{\Omega} \times \mathbb{R}^{N}$. Under the summation conventions given in the introduction, it follows from $(1.8)$ that

$$
\bar{\alpha}^{I J}(X) \xi_{I} \xi_{J} \geq \sigma_{1}|\xi|_{1}^{2}+2 \varepsilon \alpha^{i l}(X) \xi_{i} \xi_{l}+\varepsilon \sigma_{2}|\xi|_{2}^{2}
$$

Since $\left\|\alpha^{I J}\right\|_{L^{\infty}(\Omega)} \leq b$, the middle term of the right-hand side can be bounded by

$$
\begin{aligned}
\sum_{i=1}^{N_{1}} \sum_{l=N_{1}+1}^{N_{2}}\left|2 \varepsilon \alpha^{i l}(X) \xi_{i} \xi_{l}\right| & \leq \sum_{i=1}^{N_{1}} \sum_{l=N_{1}+1}^{N_{2}}\left(\frac{2 N_{1} b^{2} \varepsilon}{\sigma_{2}}\left|\xi_{i}\right|^{2}+\frac{\varepsilon \sigma_{2}}{2 N_{1}}\left|\xi_{l}\right|^{2}\right) \\
& \leq \frac{2\left(N_{2}-N_{1}\right) N_{1} b^{2} \varepsilon}{\sigma_{2}}|\xi|_{1}^{2}+\frac{\varepsilon \sigma_{2}}{2}|\xi|_{2}^{2} .
\end{aligned}
$$

Therefore,

$$
\bar{\alpha}^{I J}(X) \xi_{I} \xi_{J} \geq \frac{\sigma_{1}}{2}|\xi|_{1}^{2}+\varepsilon \frac{\sigma_{2}}{2}|\xi|_{2}^{2}
$$

for $0 \leq \varepsilon \leq \varepsilon_{0}$, with $\varepsilon_{0}=\min \left(1, \sigma_{1} \sigma_{2} /\left(4\left(N_{2}-N_{1}\right) N_{1} b^{2}\right)\right) \in(0,1]$.

Remark 2.2 If $\alpha^{i l}=0$ for all $1 \leq i \leq N_{1}$ and $N_{1}+1 \leq l \leq N_{2}$, then the conclusion of Lemma 2.1 immediately works with $\varepsilon_{0}=1$.

Let us now turn to the

Proof of Theorem 1.6. In all what follows, we assume that all the hypotheses in Theorem 1.6 are satisfied. Let $\varepsilon_{0}$ be as in Lemma 2.1 and assume that $0 \leq \varepsilon \leq \varepsilon_{0}$ and $u$ satisfies (1.9). Under the notations of Lemma 2.1, let $M$ be the elliptic operator defined by

$$
\begin{aligned}
M \phi & =\alpha^{i j}(x, y) \phi_{i j}+2 \varepsilon \alpha^{i l}(x, y) \phi_{i l}+\varepsilon \alpha^{l m}(y) \phi_{l m}+\beta^{i}(x, y) \phi_{i}+\beta^{l}(y) \phi_{l} \\
& =\bar{\alpha}^{I J}(X) \phi_{I J}+\beta^{I}(X) \phi_{I} .
\end{aligned}
$$

Let $\zeta=\zeta(X)$ be a smooth function defined in $\mathbb{R}^{N_{2}}$ such that $0 \leq \zeta \leq 1, \zeta(0)=1$ and $\zeta(X)=0$ whenever $|X| \geq 1 / 2$. Assume moreover that $\zeta$ is radial and nonincreasing; namely, there exists a smooth nonincreasing function $h$ defined on $\mathbb{R}^{+}$such that $\zeta(X)=h(|X|)$ for all $X \in \mathbb{R}^{N}$. In the sequel, the function $\zeta$ is fixed and we call $C_{0}$ its $C^{2}$ norm. Especially,

$$
\|\zeta\|_{\infty},\left\|\zeta_{I}\right\|_{\infty},\left\|\zeta_{I J}\right\|_{\infty} \leq C_{0} \text { for all } 1 \leq I, J \leq N_{2}
$$

Let $X_{0}=\left(x_{0}, y_{0}\right)=\left(x_{0,1}, \cdots, x_{0, N_{1}}, x_{0, N_{1}+1}, \cdots, x_{0, N_{2}}\right)$ be a point in $\Omega \cup \Sigma$ and let $d_{0}=$ $d\left(X_{0}, \partial \Omega \backslash \Sigma\right)$. This distance is positive whenever $X_{0}$ lies in $\Omega$ or on $\Sigma$, since $\Sigma$ is relatively open in $\partial \Omega$. 
To prove the estimates (1.10), one shall consider two cases : the first one is when the open ball $B_{d_{0}}\left(X_{0}\right)$ with center $X_{0}$ and radius $d_{0}$ is included in $\Omega$, the second case is when this ball meets $\Sigma$.

Case 1 : the open ball $B_{d_{0}}\left(X_{0}\right)$ is included in $\Omega$. Note that this case always occurs if $\Sigma=\emptyset$. Let

$$
d=\min \left(d_{0} / 2,1\right)>0
$$

let $\chi$ be the function defined by

$$
\forall X \in \mathbb{R}^{N_{2}}, \quad \chi(X)=\zeta\left(\frac{X-X_{0}}{d}\right)
$$

and let $P$ be defined in $\Omega \cup \Sigma$ by

$$
\begin{aligned}
\forall X \in \Omega \cup \Sigma, \quad P(X)=P(x, y) & =\chi^{2}(X)\left|\nabla_{x} u\right|^{2}+\lambda u^{2}(X)+\rho e^{-\kappa\left(x_{1}-x_{0,1}\right)} \\
& =\chi^{2}(X)\left(u_{k}(X)\right)^{2}+\lambda u^{2}(X)+\rho e^{-\kappa\left(x_{1}-x_{0,1}\right)},
\end{aligned}
$$

where $\lambda, \rho$ and $\kappa$ are three nonnegative real numbers to be chosen later. In order to prove the estimates (1.10) in this case 1, the key-point will be to apply a maximum principle for the function $P$.

Now let $B=B_{d}\left(X_{0}\right)$ be the open ball with center $X_{0}$ and radius $d$. One has $B \subset \Omega$ and, since $d \leq d_{0} / 2, \bar{B} \subset \overline{B_{d_{0} / 2}\left(X_{0}\right)} \subset \Omega$, and the function $u$ is bounded in $\bar{B}$ and $\overline{B_{d_{0} / 2}\left(X_{0}\right)}$, since it is locally bounded in $\Omega \cup \Sigma$. Call $B_{0}=B_{d_{0} / 2}\left(X_{0}\right), m=\inf _{B_{0}} u \leq m^{\prime}=\inf _{B} u$ and $M=\sup _{B_{0}} u \geq M^{\prime}=\sup _{B} u$. Lastly, the functions $f$ and $\nabla_{x} f$ are bounded in $B_{0} \times[m, M]$.

The function $P$ then is of class $C^{2}$ since $u$ is of class $C^{3}(\Omega)$. One is going to apply the maximum principle for the function $P$ in $B$. More precisely, one is going to check that $M P>0$ in $B$ for some well-chosen $\lambda, \rho$ and $\kappa$, in order to get that

$$
\sup _{B} P \leq \limsup _{X \rightarrow \partial B, X \in B} P(X) .
$$

In what follows, the arguments of $\zeta$ and its derivatives are $\left(X-X_{0}\right) / d$, the arguments of $f$ and its derivatives are $(X, u(X))$ and the argument of all other functions is $X$. Under the summation conventions given in the introduction, a straightforward calculation leads to

$$
\begin{aligned}
M P= & \bar{\alpha}^{I J} P_{I J}+\beta^{I} P_{I} \\
= & \bar{\alpha}^{I J}\left[\frac{2}{d^{2}}\left(\zeta_{I J} \zeta+\zeta_{I} \zeta_{J}\right) u_{k}^{2}+\frac{4}{d} \zeta\left(\zeta_{I} u_{J k}+\zeta_{J} u_{I k}\right) u_{k}\right. \\
& \left.\quad+2 \zeta^{2}\left(u_{I J k} u_{k}+u_{I k} u_{J k}\right)+2 \lambda\left(u_{I J} u+u_{I} u_{J}\right)\right]+\rho \kappa^{2} \alpha^{11} e^{-\kappa\left(x_{1}-x_{0,1}\right)} \\
& +\beta^{I}\left[\frac{2}{d} \zeta_{I} \zeta u_{k}^{2}+2 \zeta^{2} u_{I k} u_{k}+2 \lambda u_{I} u\right]-\rho \kappa \beta^{1} e^{-\kappa\left(x_{1}-x_{0,1}\right)} \\
= & 2 \zeta^{2}\left[\bar{\alpha}^{I J}\left(u_{I J k} u_{k}+u_{I k} u_{J k}\right)+\beta^{I} u_{I k} u_{k}\right] \\
& +2 \lambda u\left[\bar{\alpha}^{I J} u_{I J}+\beta^{I} u_{I}\right] \\
& +\bar{\alpha}^{I J}\left[\frac{4}{d} \zeta\left(\zeta_{I} u_{J k}+\zeta_{J} u_{I k}\right) u_{k}+\frac{2}{d^{2}} \zeta_{I} \zeta_{J} u_{k}^{2}\right] \\
& +2 \lambda \bar{\alpha}^{I J} u_{I} u_{J}+2 u_{k}^{2}\left[\frac{\bar{\alpha}^{I J} \zeta_{I J} \zeta}{d^{2}}+\frac{\beta^{I} \zeta_{I} \zeta}{d}\right] \\
& +\rho \kappa\left(\kappa \alpha^{11}-\beta^{1}\right) e^{-\kappa\left(x_{1}-x_{0,1}\right)} .
\end{aligned}
$$


By differentiating the equation (1.9) satisfied by $u$ with respect to the variable $x_{k}$ and by multiplying by $u_{k}$, it is found that

$$
\bar{\alpha}^{I J} u_{I J k} u_{k}+\beta^{I} u_{I k} u_{k}=-\bar{\alpha}_{k}^{I J} u_{I J} u_{k}-\beta_{k}^{I} u_{I} u_{k}-f_{k} u_{k}-\partial_{u} f u_{k}^{2} \text { in } \Omega .
$$

Therefore,

$$
\begin{aligned}
M P= & 2 \zeta^{2}\left[\bar{\alpha}^{I J} u_{I k} u_{J k}-\bar{\alpha}_{k}^{I J} u_{I J} u_{k}-\beta_{k}^{I} u_{I} u_{k}-f_{k} u_{k}-\partial_{u} f u_{k}^{2}\right] \\
& -2 \lambda u f \\
& +\bar{\alpha}^{I J}\left[\frac{4}{d} \zeta\left(\zeta_{I} u_{J k}+\zeta_{J} u_{I k}\right) u_{k}+\frac{2}{d^{2}} \zeta_{I} \zeta_{J} u_{k}^{2}\right] \\
& +2 \lambda \bar{\alpha}^{I J} u_{I} u_{J}+2 u_{k}^{2}\left[\frac{\bar{\alpha}^{I J} \zeta_{I J} \zeta}{d^{2}}+\frac{\beta^{I} \zeta_{I} \zeta}{d}\right] \\
& +\rho \kappa\left(\kappa \alpha^{11}-\beta^{1}\right) e^{-\kappa\left(x_{1}-x_{0,1}\right)}
\end{aligned}
$$

and

$$
\begin{aligned}
M P= & \zeta^{2}\left(\bar{\alpha}^{I J} u_{I k} u_{J k}-2 \bar{\alpha}_{k}^{I J} u_{I J} u_{k}\right) \\
& +\bar{\alpha}^{I J}\left[\zeta^{2} u_{I k} u_{J k}+\frac{4}{d} \zeta\left(\zeta_{I} u_{J k}+\zeta_{J} u_{I k}\right) u_{k}+\frac{2}{d^{2}} u_{k}^{2} \zeta_{I} \zeta_{J}\right] \\
& +2 \lambda \bar{\alpha}^{I J} u_{I} u_{J}+2 u_{k}^{2}\left[\frac{\bar{\alpha}^{I J} \zeta_{I J} \zeta}{d^{2}}+\frac{\beta^{I} \zeta_{I} \zeta}{d}-\zeta^{2} \partial_{u} f\right]-2 \zeta^{2} \beta_{k}^{I} u_{I} u_{k} \\
& -2 \lambda u f-2 \zeta^{2} f_{k} u_{k}+\rho \kappa\left(\kappa \alpha^{11}-\beta^{1}\right) e^{-\kappa\left(x_{1}-x_{0,1}\right)} .
\end{aligned}
$$

Now let us estimate each line of the right hand-side of (2.1). From Lemma 2.1, and since $\left\|\alpha^{I J}\right\|_{C^{1}(\bar{\Omega})} \leq b$ and $\alpha_{k}^{l m}=0$ for all $1 \leq k \leq N_{1}$ and $N_{1}+1 \leq l, m \leq N_{2}$, the first line of (2.1) can be estimated by

$$
\begin{aligned}
& \zeta^{2} \bar{\alpha}^{I J} u_{I k} u_{J k}-2 \bar{\alpha}_{k}^{I J} u_{I J} u_{k} \geq \zeta^{2}\left[\left(\frac{\sigma_{1}}{2} u_{i k}^{2}+\varepsilon \frac{\sigma_{2}}{2} u_{l k}^{2}\right)-2 b\left|u_{i j} u_{k}\right|-4 \varepsilon b\left|u_{i l} u_{k}\right|\right] \\
& \geq \zeta^{2}\left[\sum_{1 \leq i, j, k \leq N_{1}}\left(\frac{\sigma_{1}}{2 N_{1}} u_{i j}^{2}-2 b\left|u_{i j} u_{k}\right|\right)\right. \\
& \left.+\varepsilon \sum_{\substack{1 \leq i, k \leq N_{1} \\
N_{1}+1 \leq l \leq N_{2}}}\left(\frac{\sigma_{2}}{2 N_{1}} u_{i l}^{2}-4 b\left|u_{i l} u_{k}\right|\right)\right] \\
& \geq \zeta^{2}\left[\sum_{1 \leq i, j, k \leq N_{1}}\left\{\frac{\sigma_{1}}{2 N_{1}}\left(\left|u_{i j}\right|-\frac{2 b N_{1}}{\sigma_{1}}\left|u_{k}\right|\right)^{2}-\frac{2 b^{2} N_{1}}{\sigma_{1}} u_{k}^{2}\right\}\right. \\
& \left.+\varepsilon \sum_{\substack{1 \leq i, k \leq N_{1} \\
N_{1}+1 \leq l \leq N_{2}}}\left\{\frac{\sigma_{2}}{2 N_{1}}\left(\left|u_{i l}\right|-\frac{4 b N_{1}}{\sigma_{2}}\left|u_{k}\right|\right)^{2}-\frac{8 b^{2} N_{1}}{\sigma_{2}} u_{k}^{2}\right\}\right] \\
& \geq-\frac{2 b^{2} N_{1}^{3}}{\sigma_{1}} u_{k}^{2}-\frac{8 b^{2} N_{1}^{2}\left(N_{2}-N_{1}\right)}{\sigma_{2}} u_{k}^{2},
\end{aligned}
$$


since $0 \leq \varepsilon \leq 1$. The second line in (2.1) can be rewritten as

$$
\begin{aligned}
& \bar{\alpha}^{I J}\left[\zeta^{2} u_{I k} u_{J k}+\frac{4}{d} \zeta\left(\zeta_{I} u_{J k}+\zeta_{J} u_{I k}\right) u_{k}\right. \\
& \left.+\frac{2}{d^{2}} u_{k}^{2} \zeta_{I} \zeta_{J}\right]=\bar{\alpha}^{I J}\left[\left(\zeta u_{I k}+\frac{4}{d} \zeta_{I} u_{k}\right)\left(\zeta u_{J k}+\frac{4}{d} \zeta_{J} u_{k}\right)\right. \\
& \left.-\frac{14}{d^{2}} u_{k}^{2} \zeta_{I} \zeta_{J}\right] \\
& \geq-\frac{14 b C_{0}^{2} N_{2}^{2}}{d^{2}} u_{k}^{2} \text {. }
\end{aligned}
$$

From Lemma 2.1, and since $\beta_{k}^{l}=0$ for all $1 \leq k \leq N_{1}$ and $N_{1}+1 \leq l \leq N_{2}$, the third line in (2.1) is estimated from below as follows:

$$
\begin{aligned}
2 \lambda \bar{\alpha}^{I J} u_{I} u_{J}+2 u_{k}^{2}\left[\frac{\bar{\alpha}^{I J} \zeta_{I J} \zeta}{d}+\frac{\beta^{I} \zeta_{I} \zeta}{d}-\zeta^{2} \partial_{u} f\right] & \\
-2 \zeta^{2} \beta_{k}^{I} u_{I} u_{k} \geq & \lambda \sigma_{1} u_{k}^{2}+\varepsilon \lambda \sigma_{2} u_{l}^{2} \\
& -2 b C_{0}^{2} u_{k}^{2}\left(\frac{N_{2}^{2}}{d^{2}}+\frac{N_{2}}{d}+1\right)-2 b C_{0}^{2}\left|u_{i} u_{k}\right| \\
\geq & u_{k}^{2}\left[\lambda \sigma_{1}-2 b C_{0}^{2}\left(\frac{N_{2}^{2}}{d^{2}}+\frac{N_{2}}{d}+1+N_{1}\right)\right] .
\end{aligned}
$$

Lastly, as far as the fourth line in (2.1) is concerned, one has

$$
\begin{aligned}
-2 \lambda u f-2 \zeta^{2} f_{k} u_{k}+\rho \kappa\left(\kappa \alpha^{11}+\beta^{1}\right) e^{\kappa\left(x_{1}-x_{0,1}\right)} \geq & -2 \lambda\|u\|_{L^{\infty}(B)}\|f\|_{L^{\infty}\left(B \times\left[m^{\prime}, M^{\prime}\right]\right)} \\
& -C_{0}^{4} u_{k}^{2}-\left\|\nabla_{x} f\right\|_{L^{\infty}(\bar{\Omega} \times[m, M])}^{2} \\
& +\rho \kappa\left(\kappa \sigma_{1}-b\right) e^{-\kappa\left(x_{1}-x_{0}, 1\right)} .
\end{aligned}
$$

Putting together all the previous estimates leads to

$$
\begin{aligned}
& M P \geq\left|\nabla_{x} u\right|^{2}\left[\lambda \sigma_{1}-\frac{16 b C_{0}^{2} N_{2}^{2}}{d^{2}}-\frac{2 b N_{2} C_{0}^{2}}{d}-2 b^{2} N_{1}^{3} \sigma_{1}^{-1}\right. \\
& \left.-8 b^{2} N_{1}^{2}\left(N_{2}-N_{1}\right) \sigma_{2}^{-1}-2 b C_{0}^{2}\left(N_{1}+1\right)-C_{0}^{4}\right] \\
& -2 \lambda\|u\|_{L^{\infty}(B)}\|f\|_{L^{\infty}\left(B \times\left[m^{\prime}, M^{\prime}\right]\right)}-\left\|\nabla_{x} f\right\|_{L^{\infty}\left(B \times\left[m^{\prime}, M^{\prime}\right]\right)}^{2} \\
& +\rho \kappa\left(\kappa \sigma_{1}-b\right) e^{-\kappa\left(x_{1}-x_{0,1}\right)} \text {. }
\end{aligned}
$$

Now choose

$$
\left\{\begin{aligned}
\lambda= & \sigma_{1}^{-1}\left[\frac{16 b C_{0}^{2} N_{2}^{2}}{d^{2}}+\frac{2 b N_{2} C_{0}^{2}}{d}\right. \\
& \left.\quad+2 b^{2} N_{1}^{3} \sigma_{1}^{-1}+8 b^{2} N_{1}^{2}\left(N_{2}-N_{1}\right) \sigma_{2}^{-1}+2 b C_{0}^{2}\left(N_{1}+1\right)+C_{0}^{4}\right]>0 \\
\kappa= & \sigma_{1}^{-1}(1+b)>0 \\
\rho= & \left(\kappa e^{-\kappa}\right)^{-1}\left(2 \lambda\|u\|_{L^{\infty}(B)}\|f\|_{L^{\infty}\left(B \times\left[m^{\prime}, M^{\prime}\right]\right)}\right. \\
& \left.\quad+\left\|\nabla_{x} f\right\|_{L^{\infty}\left(B \times\left[m^{\prime}, M^{\prime}\right]\right)}^{2}+\|u\|_{L^{\infty}(B)} \operatorname{Osc}_{B}(u)\right) \geq 0 .
\end{aligned}\right.
$$

Since $\left|x_{1}-x_{0,1}\right| \leq d \leq 1$ in $B$, it then follows that $e^{-\kappa\left(x_{1}-x_{0,1}\right)} \geq e^{-\kappa}$, whence

$$
M P \geq\|u\|_{L^{\infty}(B)} \operatorname{Osc}_{B}(u) \text { in } B .
$$


If $u$ is constant in $B$, then $\nabla_{X} u=0$ in $B$ and the estimate (1.10) is immediately satisfied at $X_{0}$. Otherwise, if $u$ is not constant in $B$, then $\|u\|_{L^{\infty}(B)} \operatorname{Osc}_{B}(u)>0$, whence $M P>0$ in $B$. The maximum principle then implies that

$$
P\left(X_{0}\right) \leq \limsup _{X \rightarrow \partial B, X \in B} P(X) .
$$

Note that the maximum principle works for both $0<\varepsilon \leq \varepsilon_{0}$ (in that case, the operator $M$ is elliptic in all $(x, y)$-variables) and $\varepsilon=0$ (in that case, the matrix $\left(\bar{\alpha}^{I J}(X)\right)_{1 \leq I, J \leq N_{2}}$ is still nonnegative at any point $X \in \Omega$ ). Owing to the choice of $\zeta$, one has

$$
P\left(X_{0}\right)=\left|\nabla_{x} u\left(X_{0}\right)\right|^{2}+\lambda u^{2}\left(X_{0}\right)+\rho .
$$

On the other hand, for each point $X \in B$ such that $d / 2 \leq\left|X-X_{0}\right|<d$, one has $\chi(X)=$ $\zeta\left(\left(X-X_{0}\right) / d\right)=0$ and $\left|x_{1}-x_{0,1}\right| \leq d \leq 1$. Therefore,

$$
\limsup _{X \rightarrow \partial B, X \in B} P(X) \leq \lambda\|u\|_{L^{\infty}(B)}^{2}+\rho e^{\kappa} .
$$

From (2.3), it is found that

$$
\begin{aligned}
\left|\nabla_{x} u\left(X_{0}\right)\right|^{2} \leq & P\left(X_{0}\right)-\lambda u^{2}\left(X_{0}\right) \\
\leq & \lambda\left(\|u\|_{L^{\infty}(B)}^{2}-u^{2}\left(X_{0}\right)\right)+\rho e^{\kappa} \\
\leq & C\left(N_{1}, N_{2}, \sigma_{1}, \sigma_{2}, b\right)\left(d^{-2}+1\right) \times \\
& \times\left[\|u\|_{L^{\infty}(B)}\left(\operatorname{osc}_{B}(u)+\|f\|_{L^{\infty}\left(B \times\left[m^{\prime}, M^{\prime}\right]\right)}\right)+\left\|\nabla_{x} f\right\|_{L^{\infty}\left(B \times\left[m^{\prime}, M^{\prime}\right]\right)}^{2}\right]
\end{aligned}
$$

for some positive constant $C\left(N_{1}, N_{2}, \sigma_{1}, \sigma_{2}, b\right)$ only depending on $N_{1}, N_{2}, \sigma_{1}, \sigma_{2}$ and $b$.

Now remember that $d=\min \left(d_{0} / 2,1\right)$, where $d_{0}=d\left(X_{0}, \partial \Omega \backslash \Sigma\right)$, and $\bar{B} \subset \overline{B_{0}} \subset \Omega$. It easily follows that

$$
\begin{aligned}
\left|\nabla_{x} u\left(X_{0}\right)\right|^{2} \leq & 4 C\left(N_{1}, N_{2}, \sigma_{1}, \sigma_{2}, b\right)\left(d_{0}^{-2}+1\right) \times \\
& \times\left[\|u\|_{L^{\infty}\left(B_{0}\right)}\left(\operatorname{osc}_{B_{0}}(u)+\|f\|_{L^{\infty}\left(B_{0} \times[m, M]\right)}\right)+\left\|\nabla_{x} f\right\|_{L^{\infty}\left(B_{0} \times[m, M]\right)}^{2}\right]
\end{aligned}
$$

whenever $d_{0} / 2<1$ or $d_{0} / 2 \geq 1$.

Case 2 : the open ball $B_{d_{0}}\left(X_{0}\right)$ meets $\Sigma$. Therefore,

$$
0 \leq d\left(X_{0}, \Sigma\right)<d_{0}=d\left(X_{0}, \partial \Omega \backslash \Sigma\right) .
$$

Call $\tilde{\eta}=\min (\eta, 4)$ and $d=\min \left(\tilde{\eta} / 4, d_{0} / 4\right)>0$, where $\eta$ is given in Theorem 1.6. One shall now consider two subcases : the first one is when $d\left(X_{0}, \Sigma\right) \geq d$ and the second one is when $d\left(X_{0}, \Sigma\right)<d$.

Subcase 2- $a: d\left(X_{0}, \Sigma\right) \geq d$. In that case, one has $B=B_{d}\left(X_{0}\right) \subset \Omega$ and, since $d \leq d_{0} / 2<$ $d_{0}$, then $\bar{B} \subset \overline{B_{d_{0} / 2}\left(X_{0}\right)} \subset \Omega \cup \Sigma$, and the function $u$ is bounded in $\bar{B}$ and $\overline{B_{d_{0} / 2}\left(X_{0}\right)}$. Call $B_{0}=B_{d_{0} / 2}\left(X_{0}\right), m=\inf _{B_{0}} u \leq m^{\prime}=\inf _{B} u$ and $M=\sup _{B_{0}} u \geq M^{\prime}=\sup _{B} u$. Observe that the functions $f$ and $\nabla_{x} f$ are bounded in $B_{0} \times[m, M]$. 
By repeating the arguments of case 1 in this ball $B$ and by observing that $d \leq \tilde{\eta} / 4 \leq 1$, it is found that

$$
\begin{aligned}
\left|\nabla_{x} u\left(X_{0}\right)\right|^{2} \leq C & \left(N_{1}, N_{2}, \sigma_{1}, \sigma_{2}, b\right)\left(d^{-2}+1\right) \times \\
& \times\left[\|u\|_{L^{\infty}(B)}\left(o s c_{B}(u)+\|f\|_{L^{\infty}\left(B \times\left[m^{\prime}, M^{\prime}\right]\right)}\right)+\left\|\nabla_{x} f\right\|_{L^{\infty}\left(B \times\left[m^{\prime}, M^{\prime}\right]\right)}^{2}\right],
\end{aligned}
$$

where the constant $C\left(N_{1}, N_{2}, \sigma_{1}, \sigma_{2}, b\right)$ is the same as in (2.4). Since $\bar{B} \subset \overline{B_{0}} \subset \Omega \cup \Sigma$, it follows that

$$
\begin{aligned}
\left|\nabla_{x} u\left(X_{0}\right)\right|^{2} \leq C & \left(N_{1}, N_{2}, \sigma_{1}, \sigma_{2}, b, \eta\right)\left(d_{0}^{-2}+1\right) \times \\
& \times\left[\|u\|_{L^{\infty}\left(B_{0}\right)}\left(\operatorname{osc}_{B_{0}}(u)+\|f\|_{L^{\infty}\left(B_{0} \times[m, M]\right)}\right)+\left\|\nabla_{x} f\right\|_{L^{\infty}\left(B_{0} \times[m, M]\right)}^{2}\right],
\end{aligned}
$$

whenever $d=\tilde{\eta} / 4$ or $d=d_{0} / 2$, for some constant $C\left(N_{1}, N_{2}, \sigma_{1}, \sigma_{2}, b, \eta\right)$ which only depends on $N_{1}, N_{2}, \sigma_{1}, \sigma_{2}, b$ and $\eta$.

Subcase 2-b : $d\left(X_{0}, \Sigma\right)<d$. Call $d^{\prime}=2 d=\min \left(\tilde{\eta} / 2, d_{0} / 2\right)>0$ and $B^{\prime}=B_{d^{\prime}}\left(X_{0}\right)$. Since $d^{\prime} \leq d_{0} / 2<d_{0}$, the closed ball $\overline{B^{\prime}}$ does not meet $\partial \Omega \backslash \Sigma$, and

$$
\overline{B^{\prime} \cap \Omega} \subset \overline{B_{d_{0} / 2}\left(X_{0}\right) \cap \Omega} \subset \Omega \cup \Sigma .
$$

Hence, the function $u$ is bounded in $B^{\prime} \cap \Omega$ and $B_{0} \cap \Omega$, where $B_{0}=B_{d_{0} / 2}\left(X_{0}\right)$. Call $m=$ $\inf _{B_{0} \cap \Omega} u \leq m^{\prime}=\inf _{B^{\prime} \cap \Omega} u$ and $M=\sup _{B_{0} \cap \Omega} u \geq M^{\prime}=\sup _{B^{\prime} \cap \Omega} u$. Notice also that the functions $f$ and $\nabla_{x} f$ are bounded in $B_{0} \cap \Omega \times[m, M]$.

Furthermore, since $0 \leq d\left(X_{0}, \Sigma\right)<d\left(X_{0}, \partial \Omega \backslash \Sigma\right)$, there exists a point $Y_{0} \in \Sigma$ such that $0 \leq\left|Y_{0}-X_{0}\right|=d\left(X_{0}, \Sigma\right)$. Owing to the definition of $\eta$ in Theorem 1.6, the connected component of $B_{\eta}\left(Y_{0}\right) \backslash \Gamma$ containing $Y_{0}-r \nu\left(Y_{0}\right)$ for $r>0$ small enough is included in $\Omega$, where $\Gamma$ is the connected component of $\partial \Omega \cap B_{\eta}\left(Y_{0}\right)$ containing $Y_{0}$. Therefore, since $\left|Y_{0}-X_{0}\right|=$ $d\left(X_{0}, \Sigma\right) \leq \tilde{\eta} / 4<\eta / 2$ and $d^{\prime} \leq \tilde{\eta} / 2 \leq \eta / 2$, it follows that the connected component of $B^{\prime} \backslash \partial \Omega$ $\left(=B^{\prime} \backslash \Sigma\right.$ since $\left.d^{\prime} \leq d_{0} / 2<d_{0}=d\left(X_{0}, \partial \Omega \backslash \Sigma\right)\right)$ containing $X_{0}$ if $X_{0} \in \Omega\left(\right.$ resp. $X_{0}-r \nu\left(X_{0}\right)$ for $r>0$ small enough if $X_{0} \in \Sigma$ ) is included in $\Omega$ (see Figure 1). Furthermore, the latter still holds if $B^{\prime}$ is replaced with $B_{3 d^{\prime} / 2}\left(X_{0}\right)$.

Since $d\left(X_{0}, \Sigma\right) \leq d^{\prime} / 2$ and since $\Sigma$ is smooth (globally $C^{3}$ ) and its normal vector $\nu$ has zero $y$-components, there exists a $C^{3}$ transform wich locally straightens $\Sigma$ so that it becomes included in $\left\{x_{1}=0\right\}$, as it will be seen below. Since the vector field $\mu$ is itself globally of class $C^{3}(\Sigma)$ and satisfies $\mu(X) \cdot \nu(X) \geq \gamma>0$ on $\Sigma$, another change of variables renders $\mu=(1,0, \cdots, 0)$.

More precisely, calling $\mathcal{B}$ the projection of $B^{\prime}$ on the $x$-coordinates, there exists a $C^{3}(\overline{\mathcal{B}})$ diffeomorphism $\phi$ such that :

1) the map $\Phi: X=(x, y) \mapsto \tilde{X}=(\tilde{x}, y)=(\phi(x), y)$ maps $\overline{B^{\prime}}$ onto $\overline{\tilde{B}}$ where $\tilde{B}=B_{\tilde{d}}\left(\tilde{X}_{0}\right)$, $\tilde{X}_{0}=\Phi\left(X_{0}\right)$ and

$$
0<\theta_{1} d^{\prime} \leq \tilde{d} \leq \theta_{2} d^{\prime}
$$

for some positive constants $\theta_{1}$ and $\theta_{2}$ only depending on $\Sigma, \mu$ and $\gamma$;

2) $\Phi$ maps the connected component of $B^{\prime} \backslash \Sigma$ containing $X_{0}$ if $X_{0} \in \Omega$ (resp. $X_{0}-r \nu\left(X_{0}\right)$ for $r>0$ small enough if $X_{0} \in \Sigma$ ) onto $\tilde{B}^{-}=\tilde{B} \cap\left\{\tilde{x}_{1}<0\right\}$; in particular, the first component $\tilde{x}_{0,1}$ of $\tilde{X}_{0}$ is nonpositive (note that $\tilde{x}_{0,1}=0$ if $X_{0} \in \Sigma$ ); 


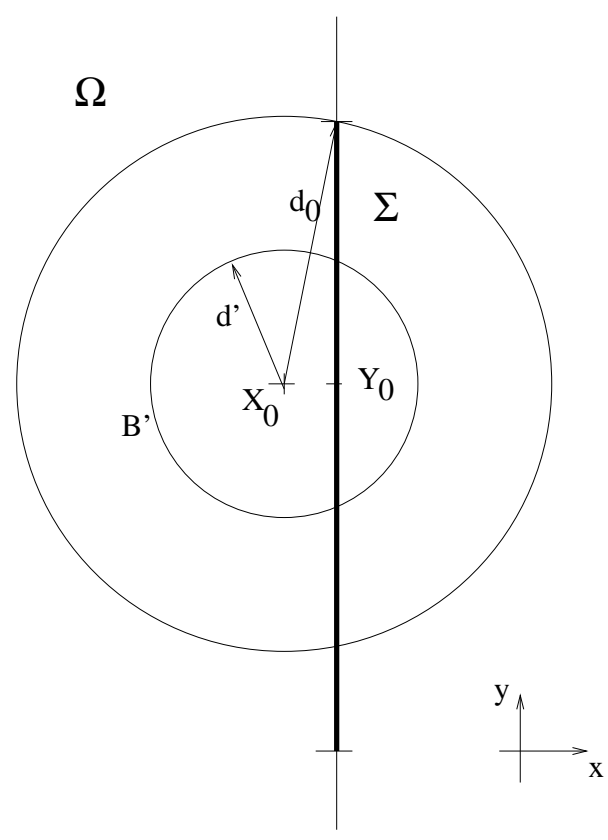

Figure 1: Subcase 2-b, with $d_{0} \leq \tilde{\eta} / 4, d=d_{0} / 4$ and $d^{\prime}=d_{0} / 2$

3) $\Phi$ maps the connected component of $B^{\prime} \cap \Sigma$ containing $Y_{0}$ onto $\tilde{\Sigma}=\tilde{B} \cap\left\{\tilde{x}_{1}=0\right\}$;

4) the norm of the derivatives of $\Phi$ in $\overline{B^{\prime}}$ and $\Phi^{-1}$ in $\bar{B}$ up to the third order only depend on $\Sigma, \mu$ and $\gamma$;

$5)$ the function $v(\tilde{X})=v(\tilde{x}, y)=u\left(\Phi^{-1}(\tilde{X})\right)=u\left(\phi^{-1}(\tilde{x}), y\right)$ is of class $C^{3}\left(\tilde{B}^{-}\right) \cap C^{2}\left(\overline{\tilde{B}^{-}}\right)$ (note that $\tilde{\Sigma} \subset \overline{\tilde{B}^{-}}$). It ranges in $\left[m^{\prime}, M^{\prime}\right]$ and satisfies an equation of the type

$$
\left\{\begin{aligned}
\tilde{\alpha}^{i j}(\tilde{x}, y) v_{i j}+2 \varepsilon \tilde{\alpha}^{i l}(\tilde{x}, y) v_{i l}+\varepsilon \tilde{\alpha}^{l m}(y) v_{l m} & \\
+\tilde{\beta}^{i}(\tilde{x}, y) v_{i}+\tilde{\beta}^{l}(y) u_{l}+\tilde{f}(\tilde{x}, y, v) & =0 \text { in } \tilde{B}^{-} \\
v_{1} & =0 \text { on } \tilde{\Sigma}
\end{aligned}\right.
$$

where $v_{i}=\partial_{\tilde{x}_{i}} v, v_{l}=\partial_{x_{l}} v$ for all $1 \leq i \leq N_{1}$ and $N_{1}+1 \leq l \leq N_{2}$, and so on for the higher-order derivatives. The functions $\tilde{\alpha}^{I J}$ and $\tilde{\beta}^{I}$ are of class $C^{1}\left(\overline{\tilde{B}^{-}}\right)$, for all $1 \leq I, J \leq N_{2}$. Actually,

$$
\tilde{\alpha}^{l m}(\tilde{X})=\alpha^{l m}(y) \text { and } \tilde{\beta}^{l}(\tilde{X})=\beta^{l}(y)
$$

for all $N_{1}+1 \leq l, m \leq N_{2}$, whence $\tilde{\alpha}^{l m}$ and $\tilde{\beta}^{l}$ do not depend on $\tilde{x}$ and the matrix $\left(\tilde{\alpha}^{l m}\right)_{N_{1}+1 \leq l, m \leq N_{2}}$ satisfies $\tilde{\alpha}^{l m}(\tilde{X}) \xi_{l} \xi_{m} \geq \sigma_{2}|\xi|_{2}^{2}$ for all $\tilde{X} \in \overline{\tilde{B}^{-}}$and $\xi \in \mathbb{R}^{N_{2}}$. The function

$$
\tilde{f}(\tilde{X}, s)=\tilde{f}(\tilde{x}, y, s)=f(x, y, s)
$$

is of class $C^{1}\left(\overline{\tilde{B}^{-}} \times \mathbb{R}\right)$. The matrix field $\left(\tilde{\alpha}^{I J}\right)_{1 \leq I, J \leq N_{2}}$ is symmetric and there exist four constants $\tilde{\sigma}_{1}>0, \tilde{b} \geq 0, C_{1} \geq 0$ and $\tilde{\varepsilon}_{0} \in(0,1]$ only depending on $N_{1}, N_{2}, \sigma_{1}, \sigma_{2}, b, \gamma, \mu$ and $\Sigma$ such that

$$
\forall \tilde{X} \in \overline{\tilde{B}^{-}}, \quad \forall \xi \in \mathbb{R}^{N_{2}}, \quad \sum_{1 \leq i, j \leq N_{1}} \tilde{\alpha}^{i j}(\tilde{X}) \xi_{i} \xi_{j} \geq \tilde{\sigma}_{1}|\xi|_{1}^{2},
$$




$$
\sum_{1 \leq i, j \leq N_{1}} \tilde{\alpha}^{i j}(\tilde{X}) \xi_{i} \xi_{j}+2 \varepsilon \sum_{\substack{1 \leq i \leq N_{1} \\ N_{1}+1 \leq l \leq N_{2}}} \tilde{\alpha}^{i l}(\tilde{X}) \xi_{i} \xi_{l}+\varepsilon \sum_{N_{1}+1 \leq l, m \leq N_{2}} \tilde{\alpha}^{l m}(\tilde{X}) \xi_{l} \xi_{m} \geq \frac{\tilde{\sigma}_{1}}{2}|\xi|_{1}^{2}+\varepsilon \frac{\sigma_{2}}{2}|\xi|_{2}^{2}
$$

for all $0 \leq \varepsilon \leq \tilde{\varepsilon}_{0}$ and $(\tilde{X}, \xi) \in \overline{\tilde{B}^{-}} \times \mathbb{R}^{N_{2}}$,

$$
\left\|\tilde{\alpha}^{I J}\right\|_{C^{1}\left(\tilde{B}^{-}\right)}+\left\|\tilde{\beta}^{I}\right\|_{C^{1}\left(\tilde{B}^{-}\right)}+\left\|\partial_{s} \tilde{f}\right\|_{L^{\infty}\left(\tilde{B}^{-} \times \mathbb{R}\right)} \leq \tilde{b}
$$

for all $1 \leq I, J \leq N_{2}$, and

$$
\left\|\nabla_{\tilde{x}} \tilde{f}\right\|_{L^{\infty}\left(\tilde{B}^{-} \times\left[m^{\prime}, M^{\prime}\right]\right)} \leq C_{1}\left\|\nabla_{x} f\right\|_{L^{\infty}\left(B^{\prime} \cap \Omega \times\left[m^{\prime}, M^{\prime}\right]\right)} .
$$

Note that even if it means decreasing $\tilde{\varepsilon}_{0}$, one can assume that $0<\tilde{\varepsilon}_{0} \leq \varepsilon_{0}$. Furthermore, the functions $\tilde{\alpha}^{i l}$ vanish if all functions $\alpha^{i l}$ vanish, for all $1 \leq i \leq N_{1}$ and $N_{1}+1 \leq l \leq N_{2}$. Therefore, as in Lemma 2.1, $\tilde{\varepsilon}_{0}$ can be chosen equal to 1 in the case where all functions $\alpha^{i l}$ vanish in $\Omega$ (that is there is no cross second-order partial derivative in (1.9)).

In what follows, $\varepsilon$ is such that $0 \leq \varepsilon \leq \tilde{\varepsilon}_{0}$. Let $\tilde{P}$ be the function defined by

$$
\forall \tilde{X} \in \overline{\tilde{B}^{-}}, \quad \tilde{P}(\tilde{X})=\zeta^{2}\left(\frac{\tilde{X}-\tilde{X}_{0}}{\tilde{d}}\right)\left|\nabla_{\tilde{x}} v\right|^{2}+\tilde{\lambda} v^{2}(\tilde{X})+\tilde{\rho} e^{-\tilde{\kappa}\left(\tilde{x}_{1}-\tilde{x}_{0,1}\right)}
$$

where $\tilde{\lambda}, \tilde{\rho}$ and $\tilde{\kappa}$ are some nonnegative real numbers. This function $\tilde{P}$ is of class $C^{2}\left(\tilde{B}^{-}\right) \cap$ $C^{1}\left(\overline{\tilde{B}^{-}}\right)$.

By repeating the arguments of case 1 , and calling

$$
\tilde{M} \tilde{P}=\tilde{\alpha}^{I J} \tilde{P}_{I J}+\tilde{\beta}^{I} \tilde{P}_{I},
$$

it easily follows that the choices

$$
\left\{\begin{array}{cc}
\tilde{\lambda}= & \tilde{\sigma}_{1}^{-1}\left[\frac{16 \tilde{b} C_{0}^{2} N_{2}^{2}}{\tilde{d}^{2}}+\frac{2 \tilde{b} N_{2} C_{0}^{2}}{\tilde{d}}\right. \\
& \left.\quad+2 \tilde{b}^{2} N_{1}^{3} \tilde{\sigma}_{1}^{-1}+8 \tilde{b}^{2} N_{1}^{2}\left(N_{2}-N_{1}\right) \sigma_{2}^{-1}+2 \tilde{b} C_{0}^{2}\left(N_{1}+1\right)+C_{0}^{4}\right]>0 \\
\tilde{\kappa}= & \tilde{\sigma}_{1}^{-1}(1+\tilde{b})>0 \\
\tilde{\rho}= & \left(\tilde{\kappa} e^{-2 \tilde{\kappa} \theta_{2}}\right)^{-1}\left(2 \tilde{\lambda}\|v\|_{L^{\infty}\left(\tilde{B}^{-}\right)}\|\tilde{f}\|_{L^{\infty}\left(\tilde{B}^{-} \times\left[m^{\prime}, M^{\prime}\right]\right)}+\left\|\nabla_{\tilde{x}} \tilde{f}\right\|_{L^{\infty}\left(\tilde{B}^{-} \times\left[m^{\prime}, M^{\prime}\right]\right)}^{2}\right. \\
& \left.+\|v\|_{L^{\infty}\left(\tilde{B}^{-}\right)} O s c_{\tilde{B}^{-}}(v)\right) \geq 0
\end{array}\right.
$$

lead to, as in $(2.2)$,

$$
\begin{aligned}
\tilde{M} \tilde{P} \geq & -2 \tilde{\lambda}\|v\|_{L^{\infty}\left(\tilde{B} \tilde{B}^{-}\right)}\|\tilde{f}\|_{L^{\infty}\left(\tilde{B}^{-} \times\left[m^{\prime}, M^{\prime}\right]\right)}-\left\|\nabla_{\tilde{x}} \tilde{f}\right\|_{L^{\infty}\left(\tilde{B}^{-} \times\left[m^{\prime}, M^{\prime}\right]\right)}^{2} \\
& +\tilde{\rho} \tilde{\kappa}\left(\tilde{\kappa} \tilde{\sigma}_{1}-\tilde{b}\right) e^{-\tilde{\kappa}\left(\tilde{x}_{1}-\tilde{x}_{0,1}\right)} \\
\geq & \|v\|_{L^{\infty}\left(\tilde{B}^{-}\right)} O s c_{\tilde{B}^{-}}(v) \text { in } \tilde{B}^{-}
\end{aligned}
$$

since $e^{-\tilde{\kappa}\left(\tilde{x}_{1}-\tilde{x}_{0,1}\right)} \geq e^{-\tilde{\kappa} \tilde{d}} \geq e^{-\tilde{\kappa} \theta_{2} d^{\prime}} \geq e^{-2 \tilde{\kappa} \theta_{2}}$ (remember that $\tilde{d} \leq \theta_{2} d^{\prime}$ from $(2.6)$ and $d^{\prime} \leq \tilde{\eta} / 2 \leq$ 2).

If $u$ is constant in $B^{\prime} \cap \Omega$, then $v$ is constant in $\tilde{B}^{-}$, whence $\nabla_{\tilde{X}} v\left(\tilde{X}_{0}\right)=\nabla_{X} u\left(X_{0}\right)=0$ and the estimate (1.10) is clearly satisfied at $X_{0}$. 
Let us now consider the case where $u$ is not constant in $B^{\prime} \cap \Omega$, that is to say that $v$ is not constant in $\tilde{B}^{-}$. The latter implies that $\|v\|_{L^{\infty}\left(\tilde{B}^{-}\right)}{ } \delta c_{\tilde{B}^{-}}(v)>0$, whence $\tilde{\rho}>0$ and $\tilde{M} \tilde{P}>0$ in $\tilde{B}^{-}$. Therefore, the function $\tilde{P}$ cannot have a local maximum at a point in $\tilde{B}^{-}$, whenever $\varepsilon$ is positive or zero.

Now assume that $\tilde{P}$ has a local maximum at a point $\tilde{Y} \in \tilde{\Sigma}$. It immediately follows that $\partial_{\tilde{x}_{1}} \tilde{P}(\tilde{Y}) \geq 0$. But since the function $v$ is of class $C^{2}\left(\tilde{B}^{-} \cup \tilde{\Sigma}\right)$ and since $v_{1}=\partial_{\tilde{x}_{1}} v=0$ on $\tilde{\Sigma}$ $\left(\subset\left\{\tilde{x}_{1}=0\right\}\right)$, it is found that $v_{I 1}=v_{1}=0$ on $\tilde{\Sigma}$ for all $2 \leq I \leq N_{2}$. Hence,

$$
\begin{aligned}
\partial_{\tilde{x}_{1}} \tilde{P}(\tilde{Y}) & =\frac{2}{\tilde{d}} \zeta\left(\frac{\tilde{Y}-\tilde{X}_{0}}{\tilde{d}}\right) \zeta_{1}\left(\frac{\tilde{Y}-\tilde{X}_{0}}{\tilde{d}}\right)\left|\nabla_{\tilde{x}} v(\tilde{Y})\right|^{2}-\tilde{\rho} \tilde{\kappa} e^{\tilde{\kappa} \tilde{x}_{0,1}} \\
& =\frac{2}{\tilde{d}} \zeta\left(\frac{\tilde{Y}-\tilde{X}_{0}}{\tilde{d}}\right) h^{\prime}\left(\left|\frac{\tilde{Y}-\tilde{X}_{0}}{\tilde{d}}\right|\right) \frac{\tilde{Y}_{1}-\tilde{x}_{0,1}}{\left|\tilde{Y}-\tilde{X}_{0}\right|} \times\left|\nabla_{\tilde{x}} v(\tilde{Y})\right|^{2}-\tilde{\rho} \tilde{\kappa} e^{\tilde{\kappa} \tilde{x} \tilde{x}_{0,1}}
\end{aligned}
$$

if $\tilde{Y} \neq \tilde{X}_{0}$, since $\zeta(X)=h(|X|)$, and $\partial_{\tilde{x}_{1}} \tilde{P}(\tilde{Y})=-\tilde{\rho} \tilde{\kappa} e^{\tilde{\kappa} \tilde{x}_{0,1}}$ if $\tilde{Y}=\tilde{X}_{0}$. Since the function $h$ is nonincreasing and since the first component $\tilde{Y}_{1}$ of $\tilde{Y}$ is zero and the first component $\tilde{x}_{0,1}$ of $\tilde{X}_{0}$ is nonpositive, it follows that $\partial_{\tilde{x}_{1}} \tilde{P}(\tilde{Y}) \leq-\tilde{\rho} \tilde{\kappa} e^{\tilde{\kappa} \tilde{x}_{0,1}}<0$ whenever $\tilde{Y}=\tilde{X}_{0}$ or not. As already emphasized, this is impossible since $\tilde{P}$ has a local maximum at $\tilde{Y}$.

Therefore, the function $\tilde{P}$ cannot have a local maximum at a point in $\tilde{B}^{-} \cup \tilde{\Sigma}$. As a consequence, it follows as in case 1 that

$$
\begin{aligned}
\left|\nabla_{\tilde{x}} v\left(\tilde{X}_{0}\right)\right|^{2} & \leq \tilde{P}\left(\tilde{X}_{0}\right)-\tilde{\lambda} v^{2}\left(\tilde{X}_{0}\right) \\
& \leq \limsup _{\tilde{X} \rightarrow \partial \tilde{B}^{-} \backslash \tilde{\Sigma}, \tilde{X} \in \tilde{B}^{-}} \tilde{P}(\tilde{X})-\tilde{\lambda} v^{2}\left(\tilde{X}_{0}\right) \\
& \leq \tilde{\lambda}\left(\|v\|_{L^{\infty}\left(\tilde{B}^{-}\right)}^{2}-v^{2}\left(\tilde{X}_{0}\right)\right)+\tilde{\rho} e^{\tilde{\kappa} \tilde{d}}
\end{aligned}
$$

Under the above notations, one has

$$
\theta_{1} d \leq \tilde{d} \leq \theta_{2} d^{\prime} \leq \theta_{2} \tilde{\eta} / 2 \leq 2 \theta_{2}
$$

with $d^{\prime}=\min \left(\tilde{\eta} / 2, d_{0} / 2\right)$ and $\tilde{\eta}=\min (\eta, 4)$. Eventually, owing to the definitions of $\tilde{\lambda}, \tilde{\kappa}, \tilde{\rho}$ and putting all the above estimates together, one gets

$$
\begin{aligned}
\left|\nabla_{\tilde{x}} v\left(\tilde{X}_{0}\right)\right|^{2} \leq & C\left(N_{1}, N_{2}, \sigma_{1}, \sigma_{2}, b, \eta, \gamma, \mu, \Sigma\right)\left(d_{0}^{-2}+1\right) \times \\
& \times\left[\|v\|_{L^{\infty}\left(\tilde{B}^{-}\right)}\left(\operatorname{osc}_{\tilde{B}^{-}}(v)+\|\tilde{f}\|_{L^{\infty}\left(\tilde{B}^{-} \times\left[m^{\prime}, M^{\prime}\right]\right)}\right)+\left\|\nabla_{\tilde{x}} \tilde{f}\right\|_{L^{\infty}\left(\tilde{B}^{-} \times\left[m^{\prime}, M^{\prime}\right]\right)}^{2}\right],
\end{aligned}
$$

for some constant $C\left(N_{1}, N_{2}, \sigma_{1}, \sigma_{2}, b, \eta, \gamma, \mu, \Sigma\right)$ only depending on $N_{1}, N_{2}, \sigma_{1}, \sigma_{2}, b, \eta$, $\gamma$, on the derivatives up to the third order of the functions representing $\Sigma$, and on the $C^{3}$ norm of $\mu$.

Furthermore, there exists a constant $C_{3}(\Phi)$, only depending on the $C^{0}\left(\overline{B^{\prime}}\right)$ norm of the gradient of $\Phi$, such that $\left|\nabla_{x} u\left(X_{0}\right)\right|^{2} \leq C_{3}(\Phi)\left|\nabla_{\tilde{x}} v\left(\tilde{X}_{0}\right)\right|^{2}$. On the other hand, since $B^{\prime}=$ $B_{d^{\prime}}\left(X_{0}\right) \subset B_{0}=B_{d_{0} / 2}\left(X_{0}\right)$, one has

$$
\left\{\begin{array}{l}
\|v\|_{L^{\infty}\left(\tilde{B}^{-}\right)}=\|u\|_{L^{\infty}\left(B^{\prime} \cap \Omega\right)} \leq\|u\|_{L^{\infty}\left(B_{0} \cap \Omega\right)} \\
o s c_{\tilde{B}^{-}}(v)=o s c_{B^{\prime} \cap \Omega}(u) \leq o s c_{B_{0} \cap \Omega}(u) \\
\|\tilde{f}\|_{L^{\infty}\left(\tilde{B}^{-} \times\left[m^{\prime}, M^{\prime}\right]\right)}=\|f\|_{L^{\infty}\left(B^{\prime} \cap \Omega \times\left[m^{\prime}, M^{\prime}\right]\right)} \leq\|f\|_{L^{\infty}\left(B_{0} \cap \Omega \times[m, M]\right)} \\
\left\|\nabla_{\tilde{x}} \tilde{f}\right\|_{L^{\infty}\left(\tilde{B}^{-} \times\left[m^{\prime}, M^{\prime}\right]\right)} \leq C_{1}\left\|\nabla_{x} f\right\|_{L^{\infty}\left(B^{\prime} \cap \Omega \times\left[m^{\prime}, M^{\prime}\right]\right)} \leq C_{1}\left\|\nabla_{x} f\right\|_{L^{\infty}\left(B_{0} \cap \Omega \times[m, M]\right)} .
\end{array}\right.
$$


Finally, the inequality (1.10) holds at $X_{0}$ and the proof of Theorem 1.6 is complete.

Acknowledgments. The authors are grateful to H. Brezis, L. Caffarelli, J.-Y. Chemin, L. Nirenberg and H. Shahgholian for stimulating discussions during the preparation of this work.

\section{References}

[1] H. Berestycki, L. Caffarelli, L. Nirenberg, Uniform estimates for regularisation of free boundary problems, In: Anal. and Part. Diff. Eq., C. Sadosky \& M. Decker eds, 1990, pp 567-617.

[2] H. Berestycki, F. Hamel, Front propagation in periodic excitable media, Comm. Pure Appl. Math. 55 (2002), pp 949-1032.

[3] L.A. Caffarelli, C.E. Kenig, Gradient estimates for variable coefficient parabolic equations and singular perturbation problems, Amer. J. Math. 120 (1998), pp 391-439.

[4] I.M. Gamba, Sharp uniform bounds for steady potential fluid-Poisson systems, Proc. Royal Soc. Edinburgh A 127 (1997), pp 479-516.

[5] I.M. Gamba, C.S. Morawetz, A viscous approximation for a 2-D steady semiconductor or transonic gas dynamic flow: existence theorem for potential flow, Comm. Pure Appl. Math. 49 (1996), pp 999-1049.

[6] D. Gilbarg, N.S. Trudinger, Elliptic partial differential equations of second order, Springer Verlag, Berlin, 1983.

[7] S. Heinze, Homogenization of flame fronts, Preprint IWR, Heidelberg, 1993.

[8] O.A. Ladyzenskaja, V.A. Solonnikov, N.N. Ural'Ceva, Linear and quasilinear equations of parabolic type, Math. Monographs, Amer. Math. Soc., 1968.

[9] O.A. Ladyzenskaja, N.N. Ural'Ceva, Équations aux dérivées partielles de type elliptique, 1968, Dunod.

[10] G.M. Lieberman, Existence of solutions to the first initial-boundary value problem for parabolic equations via elliptic regularization, In: Progress in partial differential equations: elliptic and parabolic problems, Pitman Res. Notes Math. Series, Longman Sci. Tech., 1992, pp 193-206.

[11] J.-L. Lions, Perturbations singulières dans les problèmes aux limites et en contrôle optimal, Lect. Notes Math., Springer-Verlag, 1973.

[12] J.-L. Lions, E. Magenes, Problèmes aux limites non homogènes et applications, Dunod, 1970.

[13] R. Sperb, Maximum principles and their applications, Academic Press, New York, 1981.

[14] X. Xin, Existence of planar flame fronts in convective-diffusive periodic media, Arch. Rat. Mech. Anal. 121 (1992), pp 205-233. 